\title{
TEVATRON OPTICS MEASUREMENTS USING AN AC DIPOLE*
}

\author{
R. Miyamoto, S. E. Kopp, University of Texas at Austin, Austin, TX 78712, USA \\ A. Jansson, M. J. Syphers, Fermi National Accelerator Laboratory, Batavia, IL 60510, USA
}

\section{Abstract}

The AC dipole is a device to study beam optics of hadron synchrotrons. It can produce sustained large amplitude oscillations with virtually no emittance growth. A vertical AC dipole for the Tevatron is recently implemented and a maximum oscillation amplitude of $2 \sigma(4 \sigma)$ at $980 \mathrm{GeV}$ $(150 \mathrm{GeV})$ is achieved [1]. When such large oscillations are measured with the BPM system of the Tevatron $(20 \mu \mathrm{m}$ resolution), not only linear but even nonlinear optics can be directly measured. This paper shows how to measure $\beta$ function using an AC dipole and the result is compared to the other measurement. The paper also shows a test to detect optics changes when small changes are made in the Tevatron. Since an AC dipole is nondestructive, it allows frequent measurements of the optics which is necessary for such an test.

\section{INTRODUCTION}

The Tevatron AC dipole is a short dipole magnet which produces a oscillating field and excites driven oscillations of a beam in a synchrotron (driven betatron oscillation) for optics measurements. Unlike a conventional single turn kicker/pinger magnet, it can create large sustained oscillations without decoherence and emittance growth [2]. These properties make an AC dipole useful, especially when it is used with a good beam-position-monitor (BPM) system. The recently upgraded BPM system of the Tevatron has resolution of $20 \mu \mathrm{m}$ [3].

Since the driven and natural betatron oscillations are not exactly the same[4], the difference must be considered when beam optics is measured using an AC dipole. This paper reviews the driven betatron oscillation and shows how to measure the absolute and ring-wide $\beta$ function using the Tevatron $\mathrm{AC}$ dipole. Test results of the $\mathrm{AC}$ dipole based optics measurements are also presented.

\section{DRIVEN BETATRON OSCILLATION}

The tune of an AC dipole $\nu_{\text {acd }}$ is the ratio between frequency of the AC dipole $f_{\text {acd }}$ and frequency of the beam revolution $f_{\text {rev }}: \nu_{\text {acd }}=f_{\text {acd }} / f_{\text {rev }}$. For any tunes, only their fractional parts are considered in the following. Because of the discrete sampling ${ }^{1}$, a circulating beam passing an $\mathrm{AC}$ dipole actually sees two driving tunes $\nu_{\text {acd }}$ and $1-\nu_{\text {acd }}$. The existence of two driving tunes makes a difference between the driven and natural betatron oscillation. Between

\footnotetext{
* Work supported by Fermilab Research Alliance, LLC under Contract No. DE-AC02-07CH11359 with the United States Department of Energy.

${ }^{1}$ Another example of the discrete sampling is the two betatron tunes $\nu$ and $1-\nu$ measured by a Schottky monitor.
}

two driving tunes, the one closer to the natural betatron tune $\nu$ has larger effects on a beam. Hence, the driving tune closer to the natural tune is called primary (denoted by $\nu_{d}$ ) and the other is called the secondary. For instance, in the Tevatron, frequencies of the $\mathrm{AC}$ dipole and beam revolution are $f_{\text {rev }} \simeq 47.7 \mathrm{kHz}$ and $f_{\text {acd }} \simeq 20.5 \mathrm{kHz}$ and two driving tunes are $\nu_{\text {acd }} \simeq 0.43$ and $1-\nu_{\text {acd }} \simeq 0.57$. Since the natural tune is $\nu \simeq 0.58$, the primary driving tune is $\nu_{d}=1-\nu_{\text {acd }} \simeq 0.57$ for the Tevatron AC dipole. The difference of the primary driving tune and natural tune $\delta_{d} \equiv \nu_{d}-\nu$ is an important parameter of the driven betatron motion. The excitation becomes larger when $\left|\delta_{d}\right|$ is decreased but the risk of beam losses also increases because of the finite tune spread in a beam. For hadron synchrotrons such as LHC, RHIC, and Tevatron where AC dipoles are or will be used, the typical limit is $\left|\delta_{d}\right| \simeq 0.01$.

As natural betatron oscillations, the amplitude of the driven betatron oscillation $a_{d}\left(s ; \delta_{d}\right)$ can be written with a constant of motion $A_{d}\left(\delta_{d}\right)$ and amplitude function $\beta_{d}\left(s ; \delta_{d}\right)[4]$ :

$$
a_{d}\left(s ; \delta_{d}\right)=\left|A_{d}\left(\delta_{d}\right)\right| \sqrt{\beta_{d}\left(s ; \delta_{d}\right)} .
$$

The constant of motion and amplitude function of the driven betatron oscillation are given by

$$
\begin{aligned}
& A_{d}\left(\delta_{d}\right)=\frac{\theta_{\mathrm{acd}} \sqrt{\beta_{\mathrm{acd}}}}{4} \frac{\sqrt{1-\lambda_{d}\left(\delta_{d}\right)^{2}}}{\sin \left(\pi \delta_{d}\right)} \\
& \beta_{d}\left(s ; \delta_{d}\right) \\
& =\frac{1+\lambda_{d}\left(\delta_{d}\right)^{2}-2 \lambda_{d}\left(\delta_{d}\right) \cos (2 \psi(s)-2 \pi \nu)}{1-\lambda_{d}\left(\delta_{d}\right)^{2}} \beta(s) .
\end{aligned}
$$

Here, $\lambda_{d}$ is the ratio of effects from two driving tunes:

$$
\lambda_{d}\left(\delta_{d}\right)=\frac{\sin \left(\pi \delta_{d}\right)}{\sin \left(2 \pi \nu+\pi \delta_{d}\right)},
$$

$\theta_{\text {acd }}=B_{\text {acd }} \ell_{\text {acd }} /(B \rho)$ is the maximum deflection angle by an AC dipole, $B_{\text {acd }}$ and $\ell_{\text {acd }}$ are the maximum field strength and length of the AC dipole, $(B \rho)$ is the magnetic rigidity, $\beta$ and $\psi$ are the amplitude function and phase advance of the free oscillation ${ }^{2}$, and $\beta_{\text {acd }}$ is the $\beta$ function at the $\mathrm{AC}$ dipole.

When the coherent free oscillation is excited by a single turn kicker magnet, the relative $\beta$ functions can be measured by comparing oscillation amplitudes at BPM locations. If the same analysis is applied to a driven beam, relative changes of $\beta_{d}$ are measured instead of $\beta$. From Eqs

\footnotetext{
${ }^{2}$ The phase advance is measured from the location of the AC dipole. Since the phase advance is related to the amplitude function, phase advances of the driven and natural betatron oscillations are also different [4].
} 
3 and 4 , in the first order of $\delta_{d}$, the difference of the two amplitude functions is given by

$$
\frac{\beta_{d}-\beta}{\beta} \simeq \frac{2 \pi \delta_{d}}{\sin (2 \pi \nu)} \cos (2 \psi-2 \pi \nu) .
$$

Notice this equation has the same phase dependence as the $\beta$ beat. If $\left|\delta_{d}\right|$ must be larger than 0.01 as discussed, the amplitude of Eq 5 is at least $2 \pi \delta_{d} \simeq 6 \%$. For the Tevatron whose natural tune is $\nu \simeq 0.58$, the amplitude is $2 \pi \delta_{d} / \sin (2 \pi \nu) \simeq 10 \%$. Hence, if the $\beta$ function is measured from the driven oscillations but the difference of the amplitude functions is simply ignored, more than $6 \%$ of $\beta$ beat is measured compared to the true $\beta$ function.

\section{ABSOLUTE $\boldsymbol{\beta}$ MEASUREMENT}

At two interaction points in the Tevatron, B0 and D0, there are two BPMs with no magnet inbetween. Since the beam trajectories are straight lines in such spaces, positions and angles at any locations between these two BPMs can be determined. When turn-by-turn (TBT) positions and angles of the free oscillation $x_{n}$ and $x_{n}^{\prime}$ are known, CourantSnyder parameters at the location can be calculated [5]. For instance, the $\beta$ function is given by

$$
\beta=\frac{\left\langle x_{n}^{2}\right\rangle}{\sqrt{\left\langle x_{n}^{2}\right\rangle\left\langle x_{n}^{\prime 2}\right\rangle-\left\langle x_{n} x_{n}^{\prime}\right\rangle^{2}}}
$$

where $\left\langle x_{n}^{2}\right\rangle$ is the average of $x_{n}^{2}$ and so on. If the right hand side of Eq 6 is applied to TBT data of the driven oscillation, $\beta_{d}$ at the location can be measured instead of $\beta$.

Fig 1 shows measured $\beta_{d}\left(\delta_{d}\right)$ by using Eq 6 at the B0 interaction point of the Tevatron. The $\beta$ function and the phase advance $\psi$ at the location can be determined as the fit parameters of Eq 3 to these data points. From Eqs 3 and 4 , amplitude functions $\beta_{d}$ and $\beta$ become identical when $\delta_{d}=0$. Hence, $\beta$ is equal to the value of the fit curve

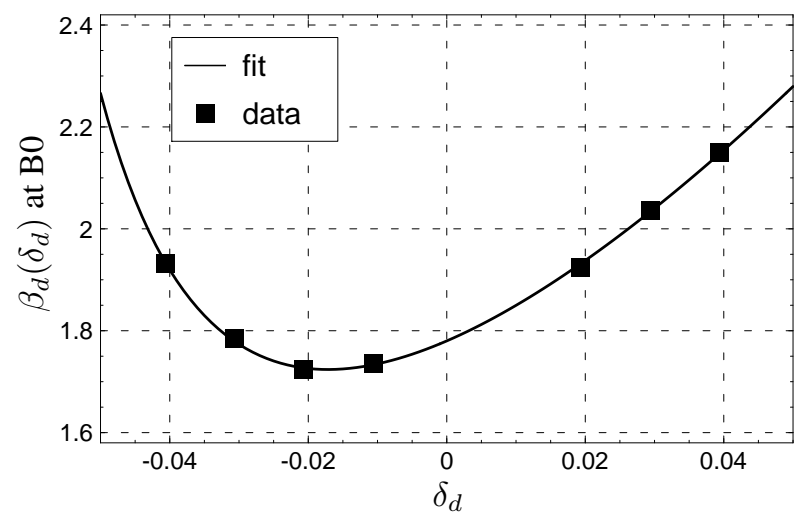

Figure 1: The relation between the amplitude function of the driven oscillation $\beta_{d}$ and the difference between the primary driving tune and natural tune $\delta_{d}$ at the $\mathrm{B} 0$ interaction point of the Tevatron. The $\beta$ function at the location is equal to $\beta_{d}\left(\delta_{d}\right) \simeq 1.78$.

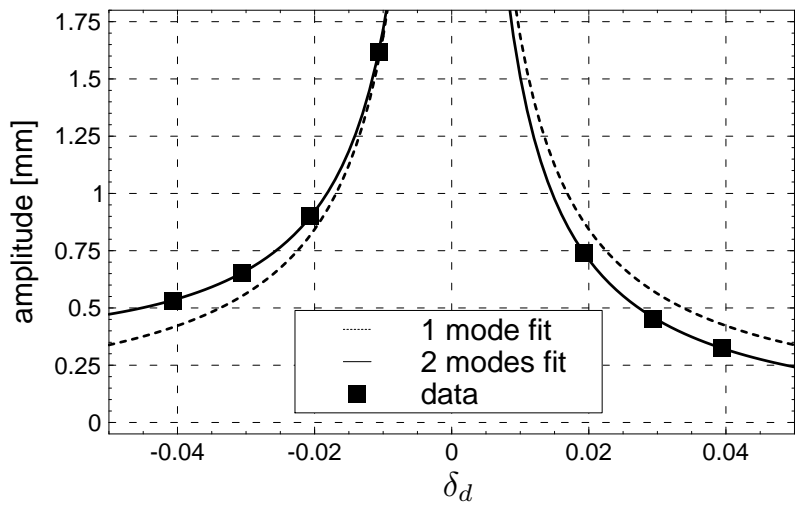

Figure 2: The relation between the oscillation amplitude of the driven betatron oscillation at one BPM and the difference of the primary driving tune and natural tune $\delta_{d}$ when the maximum deflection angle $\theta_{\text {acd }}$ is kept the same. The $\beta$ function at the location is determined up to a constant as a fit parameter. The fit becomes worse when the difference of $\beta$ and $\beta_{d}$ is ignored.

when $\delta_{d}=0$. In this way, any Courant-Snyder parameters on the collision straight sections of the Tevatron can by determined from multiple data sets of the driven betatron oscillation with different $\delta_{d}$.

\section{RING-WIDE $\beta$ MEASUREMENT}

As the absolute $\beta$ measurement in the previous section, the relative $\beta$ functions at BPMs can be also determined from multiple data sets of the driven betatron oscillation with different $\delta_{d}$. Fig 2 shows the relation between the oscillation amplitude of the driven oscillation at one BPM and $\delta_{d}$ when the maximum deflection angle $\theta_{\text {acd }}$ is kept the same. The solid line in the figure is the fit of $\mathrm{Eq} 1$ where fit parameters are $\theta_{\text {acd }} \sqrt{\beta_{\text {acd }} \beta(s)}, \psi$, and $\nu$. Since the maximum deflection angle $\theta_{\text {acd }}$ and $\beta$ at the AC dipole $\beta_{\text {acd }}$ are constants, such fit at all BPMs determine the $\beta$ functions at all BPMs up to a constant $\theta_{\text {acd }} \sqrt{\beta_{\text {acd }}}$.

The dashed line is the fit of Eq 1 with $\lambda$ set to zero by hands. Physically, it corresponds to ignore the effect from the secondary driving tune and the difference of amplitude functions between natural and driven betatron oscillations. Clearly, the model including these gives a better fit in Fig 2

The result in the previous section gives the scaling to the relative measurement in this section. The solid line in Fig 3 shows the absolute ring-wide $\beta$ functions as a combined result of two sections. The dashed line shows the measured amplitude function of the driven oscillation $\beta_{d}$ when $\delta_{d}=-0.01$. If a single data set of the driven betatron oscillation is analyzed as the free oscillation, the result looks like the dashed line. As discussed in a previous section, $\beta_{d}$ is showing $10 \%$ of beating compared to $\beta$. 


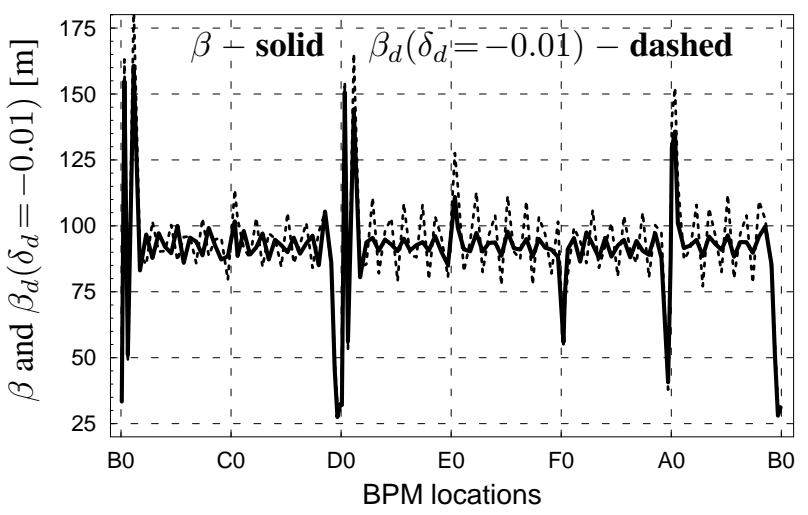

Figure 3: Comparison of measured amplitude functions $\beta$ and $\beta_{d}$. By combining two analyses of multiple TBT data sets of the driven oscillation with different $\delta_{d}, \beta$ functions at BPMs can be determined. A single data set of the driving oscillations gives $\beta_{d}$ which has $10 \%$ of beating compared to $\beta$.

\section{COMPARATIVE MEASUREMENT}

Fig 4 shows the comparison of measured $\beta$ functions of the Tevatron by two methods: the AC dipole based measurement and differential orbit measurement [6]. There is 4-5\% of scaling difference but relative differences between BPMs agree in the level of 2-3\%. The advantage of the $\mathrm{AC}$ dipole based measurement is that it is a direct measurement and independent of lattice models. Since it is a nondestructive device, multiple measurements can be done on the same beam relatively fast too.

To test the accuracy of the AC dipole based $\beta$ measurement, the $\beta$ function was intentionally changed about $10 \%$ only a part of the Tevatron and measurements were done before and after the change. Fig 5 shows the measured change of the $\beta$ function by using the AC dipole. The figure shows $10 \%$ change of the $\beta$ function can be

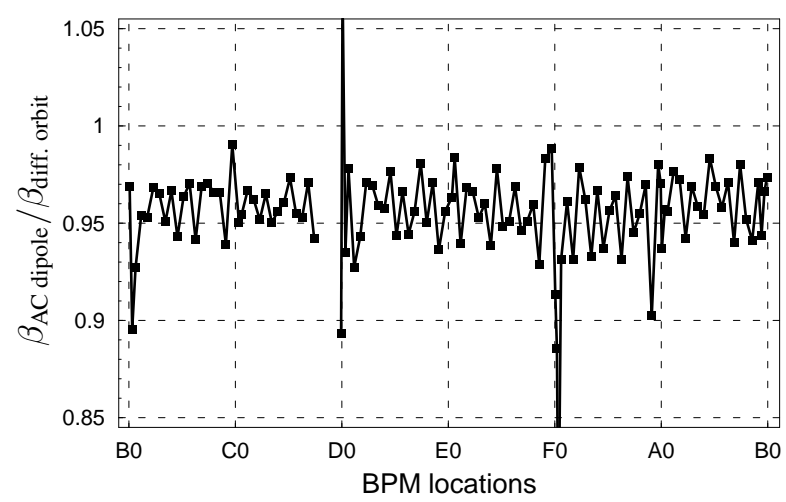

Figure 4: Comparison of the measured $\beta$ function from the AC dipole based measurement and differential orbit measurement. There is $4-5 \%$ of the scaling difference but the relative changes between BPMs agree with 2-3\% accuracy (except a few points). clearly detected. This measurement was done on the same beam whereas the re-injection and tune up would have been required if the same test were done with a conventional pinger/kicker magnet.

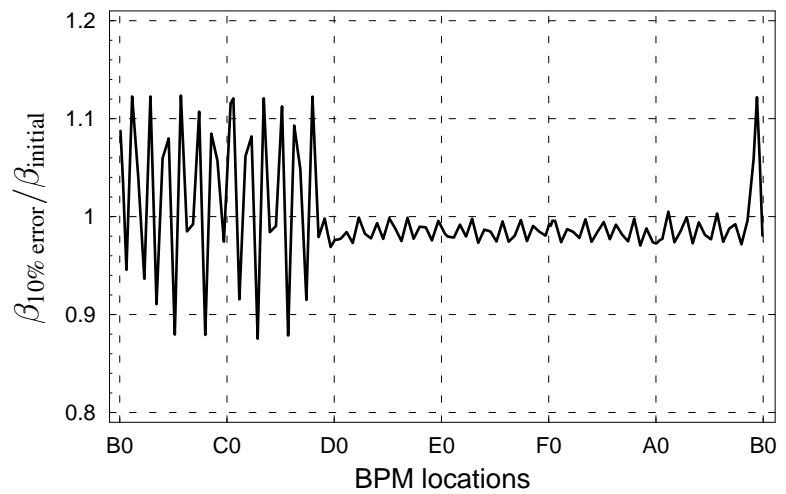

Figure 5: Test to detect changes of $\beta$ functions at BPMs. By using two quadrupole magnets, $10 \% \beta$ beat is intentionally produced from $\mathrm{B} 0$ to $\mathrm{D} 0$ and $\beta$ functions were measured before and after the change. The AC dipole based measurement can clearly detect $10 \%$ changes of the $\beta$ functions.

\section{CONCLUSION}

Driven motions of a beam excited by an AC dipole is slightly different from the natural betatron oscillation and the difference is expressed with a different amplitude functions. If the difference is simply ignored, it could affect $\beta$ function measurements more than $6 \%$ in the LHC, RHIC, and Tevatron. The absolute ring-wide $\beta$ function can be measured from multiple data sets with different driving tunes and the same field strength. In the Tevatron, the measured $\beta$ functions based on the AC dipole and differential orbit had 4-5\% scaling difference but agreed with relative changes between BPMs within 2-3\%. The AC dipole based measurement could clearly detect $10 \%$ changes of the $\beta$ functions in the Tevatron.

\section{REFERENCES}

[1] R. Miyamoto et al., "Tevatron AC Dipole System", these proceedings.

[2] M. Bai et al., "Experimental Test of Coherent Betatron Resonance Excitations", Phys. Rev. E 56, p. 6002 (1997).

[3] S. Wolbers et al., "Tevatron Beam Position Monitor Upgrade", PAC 2005, Knoxville, TN, p. 410.

[4] R. Miyamoto, "Parametrization of the Driven Betatron Oscillation”, Fermilab Report Beams-doc-2816-v1, 2007.

[5] M. J. Syphers and R. Miyamoto, "Direct Measurement of Beta-star in the Tevatron", these proceedings.

[6] V. Lebedev et al., "Measurement and Correction of Linear Optics and Coupling at Tevatron Complex", Nucl. Instrum. Methods A 558, p. 299 (2006). 